Ley, Sebastian ; Chilian, Anja; Harczos, Tamás; Kátai, András; Klefenz, Frank; Husar, Peter:

\title{
Examining basilar membrane motion of an auditory model by using tone-burst otoacoustic emissions
}

Zuerst erschienen in: Biomedical Engineering = Biomedizinische Technik. - Berlin [u.a.] : de Gruyter. - 57 (2012), Suppl. 1, Track-O, p. 285-288.

Erstveröffentlichung: $\quad$ 2012-09-06

ISSN (online): $\quad$ 1862-278X

ISSN (print): $\quad$ 0013-5585

DOI: $\quad 10.1515 / \mathrm{bmt}-2012-4145$

[Zuletzt gesehen: 2019-08-15]

„Im Rahmen der hochschulweiten Open-Access-Strategie für die Zweitveröffentlichung identifiziert durch die Universitätsbibliothek IImenau."

"Within the academic Open Access Strategy identified for deposition by Ilmenau University Library."

„Dieser Beitrag ist mit Zustimmung des Rechteinhabers aufgrund einer (DFGgeförderten) Allianz- bzw. Nationallizenz frei zugänglich."

"This publication is with permission of the rights owner freely accessible due to an Alliance licence and a national licence (funded by the DFG, German

Research Foundation) respectively." 


\title{
Examining basilar membrane motion of an auditory model by using tone-burst otoacoustic emissions
}

\author{
S. Ley ${ }^{1}$, A. Chilian ${ }^{1,3}$, T. $\operatorname{Harczos}^{2,3}$, A. Katai ${ }^{3}$, F. Klefenz ${ }^{3}$ and P. Husar ${ }^{1,3}$ \\ ${ }^{1}$ Institute of Biomedical Engineering and Informatics, Ilmenau University of Technology, Germany \\ 2 Institute for Media Technology, Ilmenau University of Technology, Germany \\ ${ }^{3}$ Fraunhofer Institute for Digital Media Technology IDMT, Ilmenau, Germany
}

\begin{abstract}
The measurement of tone-burst otoacoustic emissions (TBOAEs), which arise on the basilar membrane (BM), is a potential method for examining cochlear activity. In this contribution we consider whether TBOAEs are a suitable tool to customize the BM parameterization of an auditory model. To examine this relation, measurements of TBOAE input/output (I/O) functions were conducted in the present paper and compared to the BM I/O function of the auditory model. Latter includes models of the human basilar membrane and outer hair cells, and is simulated via wave digital filters. The results show that $\mathrm{TBOAE}$ is related to the $\mathrm{BM}$ velocity and may provide a viable tool for adjusting the parameters of the simulated BM activity.
\end{abstract}

\section{INTRODUCTION}

The measurement of otoacoustic emissions (OAEs) provides a non-invasive method to get insight into cochlear activity. In first studies Withnell and Yates used distortion product otoacoustic emissions (DPOAEs) successfully to examine $\mathrm{BM} \mathrm{I} / \mathrm{O}$ functions in guinea pigs [1]. Although this approach shows similarities between DPOAE I/O functions and BM nonlinearity, Epstein et al. suggested that the use of transientevoked otoacoustic emissions (TEOAEs) might be a more suitable tool to examine BM activity [2]. Further studies [3, 4] also indicated that TEOAEs are a suitable method to compare these measurements with psychoacoustic investigations. Epstein and Florentine demonstrated a relationship between TBOAEs and various psychoacoustic measurements and BM motion [5]. They compared TBOAEs with BM I/O functions and show a close relation between these parameters. Based on these investigations we used TBOAE I/O functions as an objective tool to examine auditory model parameters in this contribution.

\section{METHODS}

\section{A. Measurement procedure}

Otoacoustic emissions were recorded using an Etymotic ER-10C system. In order to identify the frequency range, which produces strong otoacoustic emissions, in a first investigation the ear was stimulated by clicks. The stimuli had a sound pressure level of $80 \mathrm{~dB}$ SPL (peak) and a duration of $104.2 \mu \mathrm{s}$, which corresponds to 5 samples at $48 \mathrm{ksps}$. Most of the energy is in the range between $500 \mathrm{~Hz}$ and $5 \mathrm{kHz}$. The resulting TEOAE response averaged over 800 trials was acquired with a sample rate of $48 \mathrm{ksps}$. The recordings were windowed by a $20 \mathrm{~ms}$ Hann window, which was delayed $8 \mathrm{~ms}$ related to the stimulus onset.

In consecutive investigations a Gaussian windowed tone burst with a frequency of $1 \mathrm{kHz}$ was chosen for stimulation to determine the TBOAE I/O function. The TBOAE response averaged over 800 bursts was acquired for different stimulus levels from $90 \mathrm{~dB}$ SPL (peak) downwards to $30 \mathrm{~dB}$ SPL (peak) in steps of $5 \mathrm{~dB}$ SPL. These recordings were windowed by a $20 \mathrm{~ms}$ Hann window according to [6]. The window had a delay of $15 \mathrm{~ms}$ after the beginning of the presentation, which confirms the literature [2].

\section{B. Subjects}

Six subjects, four female and two male, participated in this study. The subjects had no history of hearing difficulties and no OAE experience Their ages ranged from 22 to 27 years. A measurement session had a duration of about one hour. The participants were in a horizontal position and in a relaxed condition during the measurements.

\section{Auditory model}

The auditory model used in this study includes parts ranging from the human peripheral ear to the structures of the inner ear. The peripheral ear is represented by a model of outer and middle ear filtering, which is a linear filter having its resonant frequency at $3 \mathrm{kHz}$. It is connected to a model of nonlinear mechanical filtering, which simulates the passive cochlear hydromechanics enhanced by the active outer hair cells. It is a one-dimensional macromechanical model of the cochlea, in which the unrolled BM is divided into sections of equal length. The output of this stage represents BM 
velocity of each simulated section. The models of the peripheral ear, cochlear hydromechanics, and outer hair cells are based on [7] and [8]. The model components can be specified by differential equations, which are internally represented by equivalent electrical circuits using voltage-velocity and forcecurrent analogies. All electrical networks are simulated in the time domain using wave digital filters, because of their excellent stability properties and efficiency [9].

\section{RESULTS}

\section{A. Click evoked OAE analysis}

The click evoked OAEs of all subjects yield similar results. Figure 1 shows a spectrogram obtained with click stimuli for subject S5. The spectral density has its maximum magnitude in the frequency range about $1 \mathrm{kHz}$, which confirms the results shown in literature [2]. Based on this further investigations focused on OAE measurements using $1 \mathrm{kHz}$ tone bursts.

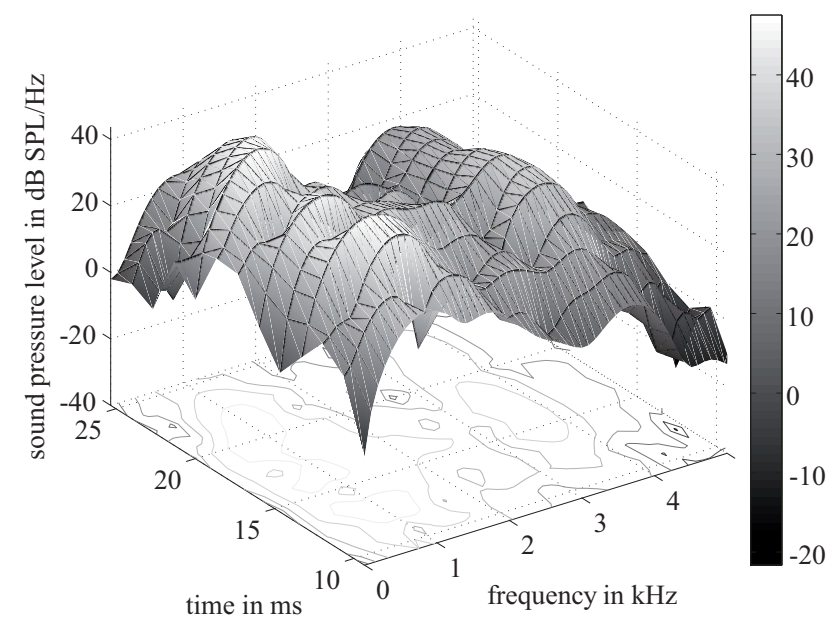

Figure 1: Click evoked OAE response of subject S5. Click stimulus was presented at $80 \mathrm{~dB} \mathrm{SPL}$ (peak) with a duration of $104.2 \mu \mathrm{s}$ and most of the energy in the frequency range between $500 \mathrm{~Hz}$ up to $5 \mathrm{kHz}$.

\section{B. TBOAE analysis}

Figure 2 shows a sample recording of windowed TBOAE responses measured in a single session for various stimulus levels. The TBOAE I/O functions were determined by calculating the RMS for each stimulus level over the corresponding windowed TBOAE response. Figure 3 shows the TBOAE I/O functions of each subject and the corresponding average noise floor, which is used to examine the signal

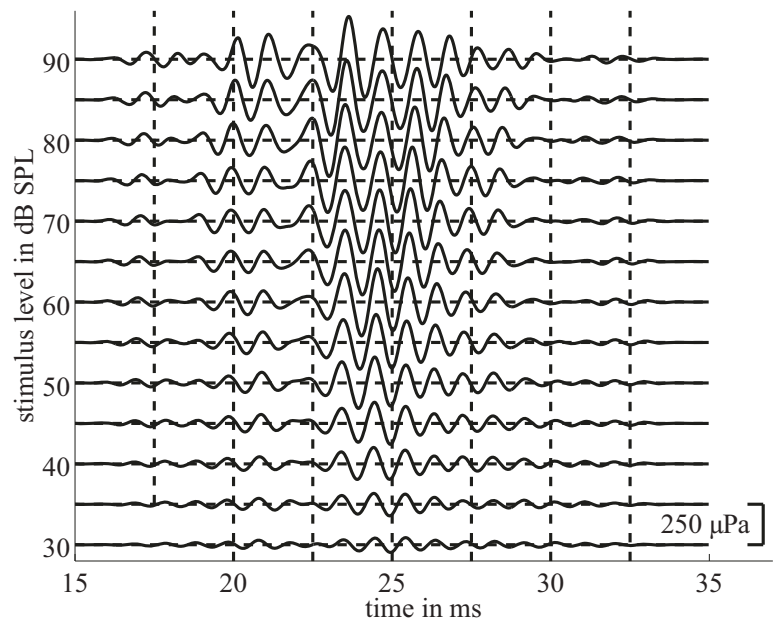

Figure 2: Individual TBOAE series, acquired for various stimulus levels from subject S5. The TBOAE responses were windowed by a $20 \mathrm{~ms}$ Hann window.

quality. For further analysis the results of subject S4 were removed, because the SNR for the lower stimulus levels is not sufficient to ensure a valid TEOAE measurement (an SNR of $6 \mathrm{~dB} \mathrm{SPL}$ and a reproducibility of more than $60 \%$ are necessary [10]). The remaining TBOAE curves show a similar non-linear shape, where the slopes are rising for lower stimulus levels and reach saturation for moderate levels. However, they exhibit interindividual differences in amplitude offsets. To compare the measurements with the simulated results of BM movement the averaged TBOAE I/O function of these subjects was computed.

\section{Comparison between TBOAE I/O and BM I/O functions}

Figure 4 shows the measured TBOAE I/O function in $\mu \mathrm{Pa}$, which is normalized to the BM velocity at a stimulus level of $30 \mathrm{~dB}$ SPL (peak) and the simulated BM velocity in $\mu \mathrm{m} / \mathrm{s}$. The input signal of the auditory model was the same burst stimulus which has also been used for determining the TBOAE I/O functions. The RMS value of the BM velocity was calculated over all sections of the BM. To improve comparability with the measured TBOAE I/O function, the response of the BM model output of each section was also multiplied by a $20 \mathrm{~ms}$ Hann window.

In an additional step, both I/O functions were analysed by using a rectangular window to investigate the influence of the window function. Figure 5 shows the RMS level of the mean TBOAE I/O function by using a rectangular window instead of the Hann window. The corresponding BM I/O function was also determined by using a rectangular window of the same length. 

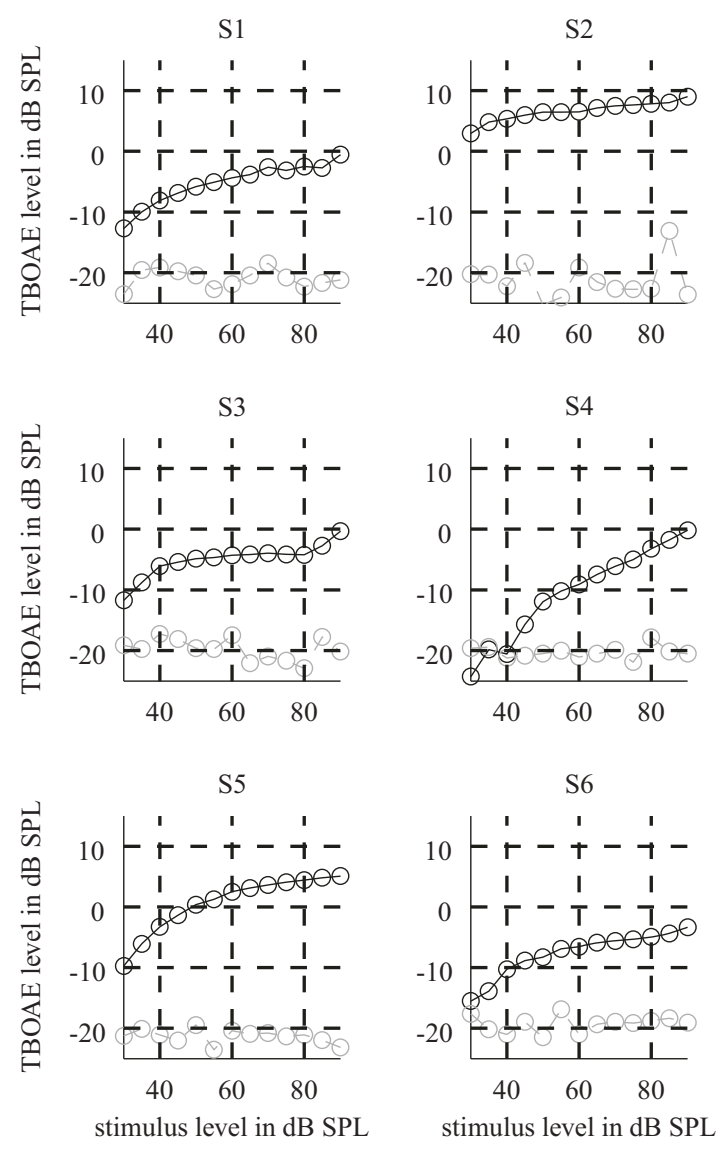

Figure 3: TBOAE I/O functions: RMS level of the TBOAE responses plotted as a function of stimulus level for each subject (black line). The grey line shows the corresponding RMS noise level.

The simulated BM I/O functions in Figure 4 and Figure 5 show differences in their amplitudes, because more signal components will be damped by using a Hann window instead of using a rectangular window. The comparison between the simulated BM I/O functions and the mean TBOAE I/O functions reveals similarities in their curve shapes. The averaged TBOAE I/O function correlates well with the simulated BM velocity for lower stimulus levels when using a Hann window. The TBOAE I/O function in Figure 5 shows a better relation to BM I/O function for high stimulus levels. The results demonstrate that the used window function influences the correlation between simulations and measurements. Another factor which also plays an important role is the time delay of the window. In the simulation BM velocity has been computed immediately after stimulus onset without any time delay. By contrast, to determine the TBOAE signal a time delay is necessary to avoid additional signal components of the stimulus signal. This also means that the early parts of the

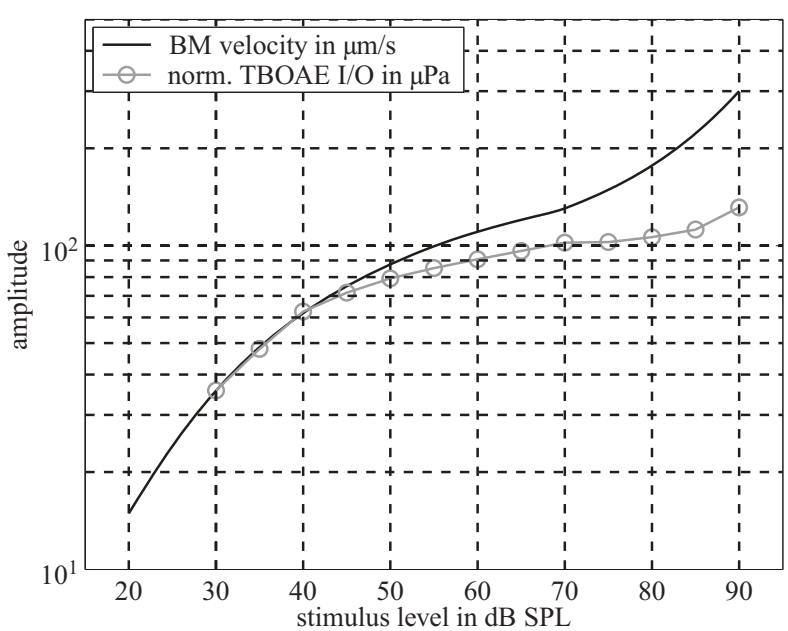

Figure 4: Simulated BM I/O function in $\mu \mathrm{m} / \mathrm{s}$ using a Hann window (black line), averaged TBOAE I/O function in $\mu \mathrm{Pa}$ also using a Hann window (grey line).

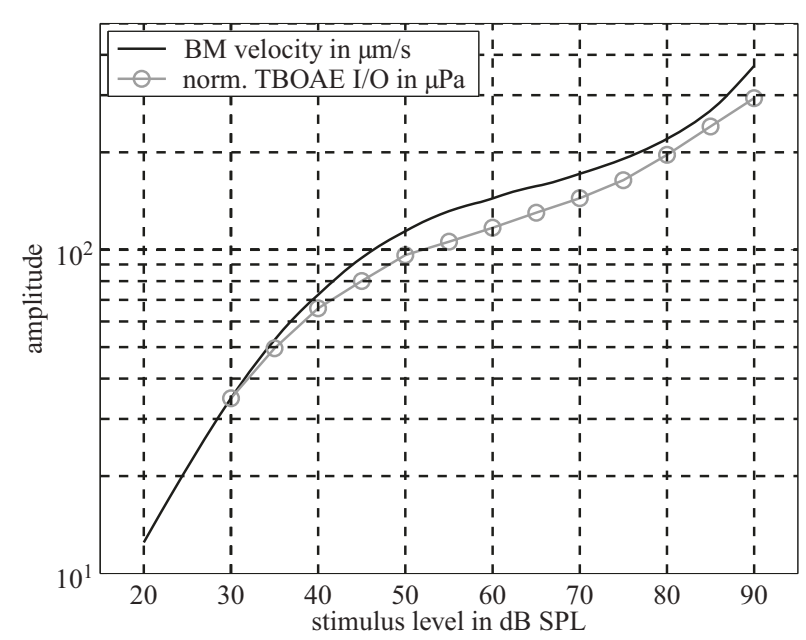

Figure 5: Simulated BM I/O function in $\mu \mathrm{m} / \mathrm{s}$ using a rectangular window (black line), averaged TBOAE I/O function in $\mu \mathrm{Pa}$ also using a rectangular window (grey line).

TBOAE response could be suppressed. This might be a reason for the deviation between simulations and measurements.

\section{CONCLUSION}

The investigations revealed a relationship between TBOAE measurements and the simulation of BM activity. The mean results of the subjects provide useful data for evaluation of the auditory model. It was shown that the results are highly influenced by the signal processing and the analysis of the acquired data. It is necessary to choose a suitable window 
function and to find a delay which show the 'true' TBOAE response and also avoids undesired signal components of the stimulus. It can be concluded that TBOAE I/O functions are a suitable tool to adjust auditory model parameters.

\section{ACKNOWLEDGEMENT}

The authors would like to thank the Institute of Biomechatronics of Ilmenau University of Technology providing the ER-10C system used in this study and especially Tobias Schmidt for valuable technical advice and support. This work was supported by the grant B514-09020 of the Thuringian Ministry of Education, Science and Culture.

\section{REFERENCES}

1. Withnell R, Yates G. Onset of basilar membrane non-linearity reflected in cubic distortion tone input-output functions. Hearing research. 1998;123:87-96.

2. Epstein M, Buus S, Florentine M. The effects of window delay, delinearization, and frequency on tone-burst otoacoustic emission input/output measurements. Journal of the Acoustical Society of America. 2004;116:1160-1167.

3. Norton S J, Neely S T. Tone-burst-evoked otoacoustic emissions from normal-hearing subjects. Journal of the Acoustical Society of America. 1987;81:1860-1872.

4. Neumann J, Uppenkamp S, Kollmeier B. Relations between notchednoise supressed TEOAE and psychoacoustical critical bandwidth. Journal of the Acoustical Society of America. 1997;101:2778-2788.

5. Epstein M, Florentine M. Inferring basilar-membrane motion from tone-burst otoacoustic emissions and psychoacoustic measurements. Journal of the Acoustical Society of America. 2005;117:263-274.

6. Basilar-membrane activity and loudness. 2006.

7. Baumgarte F. A physiological ear model for the emulation of masking. ORL J Otorhinolaryngol Relat Spec. 1999;61:294-304.

8. Baumgarte F. Ein Psychophysiologisches Gehörmodell zur Nachbildung von Wahrnehmungsschwellen für die Audiocodierung. $\mathrm{PhD}$ thesis Faculty of Electrical Engineering and Computer Science, University of Hannover, Germany 2000.

9. Fettweis A. Wave digital filters: Theory and practice. Proc. IEEE. 1986;74:270-327.

10. Hoth S, Polzer M, Neumann K, Plinkert P. TEOAE amplitude growth, detectability, and response threshold in linear and nonlinear mode and in different time windows. International journal of audiology. 2007;46:407-418.

\section{Author: Sebastian Ley}

Institute: Institute of Biomedical Engineering and Informatics

Street: Gustav-Kirchhoff-Str. 2

City: Ilmenau

Country: Germany

Email: sebastian.ley@tu-ilmenau.de 